\title{
A DYNAMICAL COEFFICIENT MECHANICS MODEL FOR FATIGUE CRACK GROWTH UNDER CONSTANT AMPLITUDE LOADING
}

\author{
Z. F. Liu, L. X. Gu, Z. Y. Xu
}

School of Civil Engineering and Transportation, South China University of Technology, Guangzhou, 510640, China(zsufangzi@163.com)

\begin{abstract}
Almost all load bearing components usually experience variable amplitude loading (VAL) rather than constant amplitude loading (CAL) during their service lives. Although many models have been proposed on this subject, but life prediction under these complex situations is still under constant improvement. The present study aims at evaluating residual fatigue life under CAL due to application of the inertial effect coefficient by adopting a dynamical coefficient mechanics (DCM) model. The proposed model was illuminated based on static fracture mechanics and the correlative problems of dynamic fracture mechanics were changed into ones of linear elastic fracture mechanics (LEFM) by d'alembert's principle. A new expression for the fatigue crack propagation (FCP) rate has thus been derived. The expression was verified by using couple samples from published experimental results and is in good agreement with the test results.
\end{abstract}

Keywords: Dynamical coefficient mechanics model, Fatigue crack propagation rate, Constant amplitude loading, Inertial effect coefficient.

\section{INTRODUCTION}

Fatigue is an important failure mode for many structures, and it has been reported that $80-90 \%$ of failures in steel structures are related to fatigue and fracture[1,2]. The fatigue strength of a component or structure can be significantly reduced by the presence of a crack or any other sharp discontinuities. Thus it is important for assessing the remaining fatigue life as precisely as possible to ensure safety, and which attracts much attention from many investigators in many countries[3,4]. Hence, reliable estimation of fatigue crack propagation and residual life prediction are essential so that the component can be timely serviced or replaced.

Nowadays, two groups of mechanistic models for fatigue crack propagation are generally accepted: the model of Laird and the model of Neumann. Laird[5]proposed a model in which the fatigue crack extension could be understood as crack tip blunting (during loading) and crack tip resharpening (during unloading). Modifications of this model have been proposed by Tomkins et al., Krasowsky et al.and Wanhill[6-8], however, the concept of multiple slip band formation resulting in the blunting at the crack tip is always present (ductile-like crack extension). Neumann[9,10] approached the problem incorporating the slip processes differently. He proposed a model in which crystallographic cleavage is also involved (brittle-like type crack extension). Many other crack propagation models can be consedered as modifications of these two, and each model has its own capabilities and 
limitations as discussed in several literature[11,12]. Because of the complexity, large ambiguities and disagreements, and also lack of proper understanding of the mechanism of retardation, no fundamental and universally accepted model is available that would include all the mechanisms and could be applied to all materials. From engineering point of view, retardation models should be calibrated by experimental data fitting as recommended by Broek[13]. Therefore, for successful implementation of life prediction methodologies to the design and in-service operation of structures subjected to variable amplitude loading, empirical models are under constant improvement.

In the present study, attempts were made to develop a DCM model for FCP under CAL based on static fracture mechanics with thinking about the inertial effect coefficient and its impact to crack tip, while the correlative problems of dynamic fracture mechanics were changed into ones of linear elastic fracture mechanics by d'alembert's principle[14]. A FCP rate expression, which reveals the correlation between FCP and intrinsic properties of the materials, nominal stress intensity factor range, an applied stress ratio, is derived. The FCP rate expression was examined objectively by the test results from literature. Finally, the DCM model and the effects of various parameters on the FCP rate expression were discussed.

\section{A DCM MODEL FOR FATIGUE CRACK GROWTH UNDER CAL}

\subsection{The inertial effect to fatigue crack caused by alternating loading}

If any particle $A$ is vibrating around its equilibrium position in the frequency of $\omega$, then its displacement is $\mu_{y}=A_{v} \sin \omega t$, its acceleration is $\ddot{\mu}_{v}=-A_{v} \omega^{2} \sin \omega t$, its inertial force is $P_{v}=m_{A} A_{v} \omega^{2} \sin \omega t$. When the full-court inertial force serve as an additional loading on the component, it can be formally changed into a statics problem by using the d'alembert's principle. At the same time, its effect to the crack tip can be identified as a crack tip singularity field according the LEFM. So we can use $K_{A}$ serve as the maximum stress intensity factor of the inertial force field. Then the maximum of $K_{B}$ can be described as follows:

$$
\begin{gathered}
K_{B}=K_{A}+K_{a}=m K_{a} \quad(m>1) . \\
K_{a}=\sigma_{a} \sqrt{\pi a_{n}} .
\end{gathered}
$$

When $K_{a}=K_{t h}$, then $K_{B}=K_{C}+K_{A}$. That's why the fatigue crack will expand slowly when $K_{B}$ is less than $K_{C}$. According d'alembert's principle, we can give out the following equation:

$$
m K_{t h}=K_{C}
$$




\subsection{Fatigue crack growth theory under symmetric CAL}

Fatigue crack growth is consisted of crack initiation, crack growth, crack arrest in every cycle as shown in Figure 1. The mechanics model of fatigue crack growth which is under the condition of symmetric CAL, can be outlined as follows:

$$
\begin{gathered}
\sigma_{y}=\sigma_{a} \sin \omega t . \\
K_{M}=K_{a} \sin \omega t . \\
K_{a}=\sigma_{a} \sqrt{\pi a_{n}} . \\
\alpha=\arcsin \frac{K_{M}}{\sigma_{a} \sqrt{\pi a_{n}}} \quad(0 \leq \alpha \leq \pi) .
\end{gathered}
$$

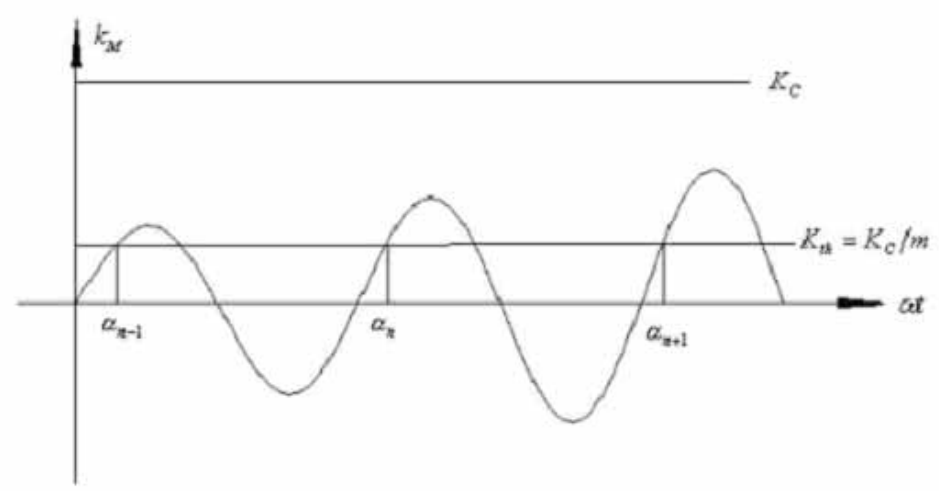

Figure 1 Schematic of fatigue crack growth condition

According Figure 1, we know $\Delta \alpha_{n}=\alpha_{n+1}-\alpha_{n}$. Then the mathematical formulation and computational procedure of fatigue crack growth can be outlined as follows:

$$
\begin{gathered}
K_{a} \sin \alpha_{n}=K_{t h} . \\
\frac{d K_{a}}{d a} \cdot \frac{d a}{d n} \sin \alpha_{n}+K_{a} \cdot \frac{d \alpha_{n}}{d n} \cos \alpha_{n}=0 \\
\frac{d a}{d n}=-\frac{K_{a} \operatorname{ctg} \alpha_{n}}{d K_{a} / d a} \times \frac{d \alpha_{n}}{d n} . \\
\alpha_{n}=\alpha(n)=c_{0}+c_{1} n+c_{2} n^{2}+\ldots . \\
\frac{d \alpha_{n}}{d n}=c_{1}+2 c_{2} n+\ldots .
\end{gathered}
$$

We all know $d a / d n$ only relates with the fracture dynamics parameters, that is to say, $d \alpha_{n} / d n$ has no relation to $n$. Meanwhile, $c_{1}$ is subtractive with monotone increasing $a$ and 
monotone decreasing $\alpha_{n}$. So $d \alpha_{n} / d n$ is determined by linear items in Eq. (12). Then we can deduce the FCP expression of infinite board with central crack as below:

$$
\begin{gathered}
a_{n}=\frac{K_{t h}^{2}}{\sigma_{a}^{2} \pi \sin ^{2}\left(c_{0}+c_{1} n\right)} . \\
c_{0}=\arcsin \frac{K_{t h}}{\sigma_{a} \sqrt{\pi a_{0}}} .
\end{gathered}
$$

The corresponding FCP rate expression is as follows:

$$
\frac{d a}{d N}=-\frac{2 c_{1} K_{t h}^{2} \cos \alpha_{n}}{\sigma_{a}^{2} \pi \sin ^{3} \alpha_{n}}=-\frac{2 c_{1} K_{a_{n}}^{2} \sqrt{K_{a_{n}}^{2}-K_{t h}^{2}}}{\sigma_{a}^{2} \pi K_{t h}} .
$$

\subsection{Fatigue crack growth theory under asymmetric CAL}

According above deducing procedure, the mechanics model of fatigue crack growth which is under the condition of asymmetric CAL, can be outlined as follows:

$$
\begin{gathered}
\sigma=\sigma_{m}+\sigma_{a} \sin \omega t . \\
m K_{t h r}=K_{C}-K_{m} . \\
K_{t h r}=K_{t h}\left(1-\frac{K_{m}}{K_{C}}\right)
\end{gathered}
$$

Then we can deduce the FCP rate expression of infinite board with central crack under asymmetric CAL as below:

$$
\begin{gathered}
C=-\frac{C_{1}}{2 \pi \sigma_{a}^{2}} . \\
a=a_{0}+\sum_{i=1}^{i=n}\left(\frac{d a}{d N}\right)_{i} . \\
2 K_{a}=\Delta K_{a}=K_{\max }-K_{\min } . \\
2 K_{t h}=\Delta K_{t h}=\frac{2 K_{C}}{m} . \\
2 K_{t h r}=\Delta K_{t h r}=\Delta K_{t h}\left(1-\frac{K_{m}}{K_{C}}\right) .
\end{gathered}
$$




$$
\begin{gathered}
K_{m}=K_{a} \cdot \frac{1+R}{1-R} . \\
\frac{d a}{d N}=C \Delta K_{a}^{2} \sqrt{\left(\Delta K_{a} / \Delta K_{t h r}\right)^{2}-1}=-2 c_{1} a \sqrt{\left(K_{a} / K_{t h r}\right)^{2}-1} .
\end{gathered}
$$

$c_{1}$ expresses the variation trend of dehiscent angle, $\Delta K_{a}$ expresses the driving force of fatigue crack growth, $\Delta K_{\text {thr }}$ expresses the resisting force of fatigue crack growth, $R$ expresses the effect of stress ratio, which were analyzed by Xu et al.[15].

\section{VERIFICATION OF THE DCM MODEL FOR FATIGUE CRACK GROWTH}

In order to assess the utility of the DCM model, the theoretical FCP analysis under constant and asymmetric amplitude loading were predicted and compared with those obtained from a set of CT specimens' experimental data which were reported by Zhang et al. [16]. Figures 2 clearly shows that there is a good agreement between the predicted value and test value when every phase has data in the FCP process. In other words, there is uncertainty only with the data of crack growth phase. That is to say, the DCM model can not only figure out the fatigue life of specimens quickly and accurately, but also analyze the whole fatigue crack growth process well just with finite test data in every phase of the FCP process.
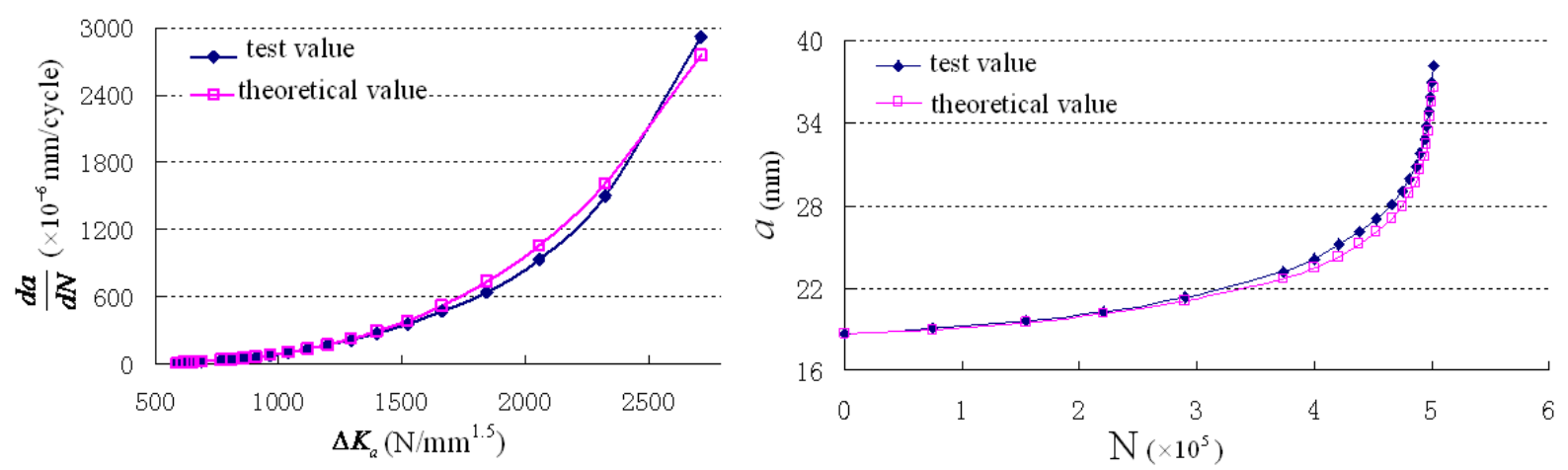

Figure 2: Comparison between predicted and measured FCP rates and crack length(test parameters, $C=5.8743 e-11 \mathrm{~m}^{4} /\left(\right.$ cycle $\left.\left.\cdot \mathrm{N}^{2}\right), K_{C}=5052.5 \mathrm{~N} / \mathrm{mm}^{1.5}, K_{t h}=312.5 \mathrm{~N} / \mathrm{mm}^{1.5}\right)$

\section{CONCLUSIONS}

Based on static fracture mechanics with thinking about the inertial effect coefficient and its impact to crack tip, a DCM model for fatigue crack growth under CAL is developed in this paper. The following preliminary conclusions could be drawn:

(1) According to the principles of static fracture mechanics and linear elastic fracture mechanics, a dynamical coefficient $m$ is suggested. The inertial effect to fatigue crack caused by alternating loading explains the mechanism of fatigue crack propagates below $K_{C}$.

(2) The DCM model can clearly describe the whole process of fatigue crack growth and calculate the residual fatigue life getting the values of $K_{C}, c_{1}$ and $m$ by some test data in 
the three phases of fatigue crack growth. This is of practical importance for engineering applications.

(3) Based on the fracture model, a new expression for the FCP rate under CAL, which reveals the correlation between the FCP rate and intrinsic properties of component's materials and imposed load, is derived for CCT specimens as follows: $d a / d N=-2 c_{1} K_{a_{n}}^{2} \sqrt{K_{a_{n}}^{2}-K_{t h}^{2}} / \sigma_{a}^{2} \pi K_{t h}$ or $d a / d N=-2 c_{1} a \sqrt{\left(K_{a} / K_{t h r}\right)^{2}-1}$.

(4) The new expression is verified objectively by comparisons with test data taken from literature. It shows a fairly good agreement with the test data. The new expression can provide a simple engineering means to analyze the fatigue crack growth rule and predict fatigue life under CAL.

\section{Acknowledgements}

Supports from National Natural Science Foundation of China under grant number 50978105 are greatly acknowledged.

\section{REFERENCES}

[1] Moses F., Schilling C.G., Raju K.S., "Fatigue evaluation procedures for steel bridges”. National Cooperative Highway Research Program (NCHRP) Rep. No. 299, Transportation Research Board, Washington, D.C. 1987.

[2] Li, W., Cheung, M.M.S., “Probabilistic fatigue and fracture analysis of steel bridges”. $J$ Struct Saf. 23, 245-62, 2003.

[3] Kuntz P., Kulak G.L., "Remaining life of existing steel bridges”. In: 3rd international symposium on steel bridges. October 30 to November 1, Rotterdam, Netherland, 1996.

[4] Zhou, Y.E., "Assessment of bridge remaining fatigue life through field strain measurement”. ASCE J Bridge Eng. 11(6), 737-44, 2006.

[5] Laird C., "The influence of metallurgical structure on the mechanisms of fatigue crack propagation”. ASTM STP. 415,131,1967.

[6] Tomkins B., Biggs W.D., J Mater Sci. 4, 532-8, 1969.

[7] Krasowsky A.J., Stepanenko V.A., “A quantitative stereoscopic fractographic study of the mechanism of fatigue crack propagation in nickel”. Int J Fract.15, 203-15, 1979.

[8] Wanhill R.J.H., "Microstructural Influences on Fatigue and Fracture Resistance in High Strength Structural Materials”. Engineering Fractrue Mechanics. 10, 337-357, 1978.

[9] Neumann P., “ New experiments concerning the slip processes at propagating fatigue cracks”. Acta Metall. 22, 1155-65, 1974. 
[10] Neumann P., Acta Metall. 22, 1167-78, 1974.

[11] Sadananda K, Vasudevan A. K, Holtz R. L, Lee E. U., “Analysis of overload effects and related phenomenon”. Int J Fatigue. 21, S233-46, 1999.

[12] Murthy A. R. C., Palani G. S., Iyer N. R., "State-of-the -art review on fatigue crack growth analysis under variable amplitude loading”. IE(1) J-CV. 85, 118-29, 2004.

[13] Broek D., “The practical use of fracture mechanics”. 1988.

[14] Kanninen, Melvin F., Popelar, C.H., 《Advanced fracture mechanics》 . Oxford Universtity Press, 563pages, 1985.

[15] Xu, Z.Y., “The Investigation of Fatigue Crack growth by Mechanics Approcach”. Modern Mathematics and Mechanics-VIII, 11, 306-310, 2000.

[16] Zhang, M., "The test methods analysis of threshold stress intensity factor of fatigue crack growth”. Transaction of Nan Jing Aeronautics college. 10, 301-306, 1992. 